\title{
Mineração
}

\section{Avaliação dos danos causados pelo desmonte com explosivos no contorno de galerias subterrâneas a partir de um modelo baseado em medidas de velocidade de partícula}

\author{
João Carlos Ribeiro Monteiro da Silva \\ Engenheiro Geólogo e Engenheiro de Minas. Mestre em Engenharia Mineral, DEMIN/UFOP \\ E-mail:joaocarlos@sequencia.eng.br
}

Valdir Costa e Silva

Universidade Federal de Ouro Preto - UFOP. Programa de Pós-Graduação em Engenharia Mineral - DEMIN

E-mail:valdir@demin.ufop.br

\begin{abstract}
Resumo
A ação das ondas de choque de detonações realizadas para a abertura de galerias subterrâneas induz danos ao maciço rochoso remanescente. Esse trabalho tem por objetivo avaliar a extensão desses danos, aplicando uma metodologia baseada no modelo de Holmberg e Persson, ao caso da mina subterrânea de Agalmatolito da LAMIL em Pará de Minas, MG. A partir de medidas de velocidade de partícula e das velocidades de propagação da onda primária, determinaram-se limites de vibração, acima dos quais são esperados danos. Utilizando a equação de atenuação das vibrações no campo próximo, desenvolvida para o local, calcularam-se os raios de duas zonas de dano em torno de duas classes de maciço: 0,24 m (raio de dano crítico) e 1,64 m (raio de dano mínimo) para o Metariolito; e 1,29 m e 1,59 m, respectivamente, para o Agalmatolito. Confrontando-se os resultados obtidos com aqueles constantes na literatura, verificou-se consistência com os valores encontrados para rochas semelhantes. Uma comparação da posição efetiva da superfície escavada com aquela prevista pelo modelo apresentou um erro de estimativa médio de $0,06 \mathrm{~m}$.
\end{abstract}

Palavras-chave: explosivos, vibração, desmonte, mina subterrânea, dano.

\begin{abstract}
The effect of shock waves from blasts in underground openings, induces damage in the adjacent rock-mass. This paper has the objective to evaluate the extension of this damage, applying a Holmberg and Persson's model-based methodology to the case of LAMIL Agalmatolite underground mine in Pará de Minas. Monitoring vibration particle velocities and $P$-wave propagation velocities, vibration limits were determined, above which, damage is expected to occur. Using the local near field scaled weight law, two radii of the damage zones around two classes of rock-mass were calculated: 0,24 $\mathrm{m}$ (critical damage radius) and $1,64 \mathrm{~m}$ (minimum damage radius) for Metarhyolite; and $1,29 \mathrm{~m}$ and $1,59 \mathrm{~m}$, respectively, for Agalmatolite. Facing measured data with existing literature showed consistency with the values found for similar rocks. A comparison of the surveyed position of the excavated surface with that predicted by the model indicated an estimated medium error of $0,06 \mathrm{~m}$.
\end{abstract}

Keywords: explosives, vibration, blast, underground mine, damage. 


\section{Introdução}

Muitas vezes, ao se buscar uma fragmentação satisfatória na detonação de galerias de minas subterrâneas, pode-se induzir no maciço adjacente um carregamento excessivo de tensões devido à ação das ondas de choque. Isto pode gerar novas fraturas e/ou movimentação e abertura de fraturas preexistentes.

A sobrequebra (overbreak) - quebra ou significativa redução da qualidade geomecânica da rocha além do perímetro projetado da escavação - deve ser minimizada, evitando-se, entre outros, os seguintes efeitos indesejáveis: aumento de tempo de remoção de "chocos" e atraso no carregamento do material detonado; gasto com instalação de suportes; piora nas condições de segurança e ventilação; aumento da diluição do minério.

Essa pesquisa teve como objetivo avaliar a influência das detonações de produção, nas condições de estabilidade da rocha remanescente em torno das galerias da mina subterrânea de agalmatolito da Lamil, lavrada pelo método de câmaras e pilares em rocha considerada de média qualidade geomecânica.

\section{Metodologia empregada}

\subsection{Critérios de avaliação da extensão das zonas de dano}

Para se avaliar a extensão das zonas de rocha danificada pelas detonações no contorno das galerias e câmaras abertas para lavra do agalmatolito, foi utilizada a metodologia proposta por Ryan e Harris (2000), que chamaram de Critério Sueco o critério de Holmberg-Persson (Persson et al., 1994), o qual define uma zona de dano crítico onde ocorre a ruptura da rocha por tração com criação de novas fraturas, sendo o mesmo expresso pela equação:

$\mathrm{PPV}_{\text {crítica }}=\frac{\sigma_{\mathrm{T}} \cdot \mathrm{V}_{\mathrm{p}}}{\mathrm{E}}$

Onde:

$\mathbf{P P V}_{\text {crítica }}=$ Velocidade máxima de partícula suportada pela rocha antes do rompimento por tração (mm/s).

$\boldsymbol{\sigma}_{\mathrm{T}}=$ Resistência à tração da rocha (MPa).

$\mathbf{V}_{\mathbf{p}}=$ Velocidade de propagação da onda primária $(\mathrm{m} / \mathrm{s})$.

$\mathbf{E}=$ Módulo de Young (GPa).

Para que se possa avaliar também a extensão máxima da zona de danos, onde fraturas preexistentes na rocha podem se propagar e dilatar sob a influência de níveis relativamente baixos de vibração, Ryan e Harris (2000) adotaram o critério do CANMET (Calder, 1977), o qual, com as modificações sugeridas por Silva (2004), é expresso pela equação:
$\mathrm{PPV}_{\text {mínima }}=\frac{0,021 \sigma_{1}}{\mathrm{~V}_{\mathrm{p}} \cdot \rho_{\mathrm{R}}}$

onde:

$\mathbf{P P V}_{\text {mínima }}=$ Velocidade mínima de partícula causadora de extensão e dilatação de fraturas preexistentes $(\mathrm{mm} / \mathrm{s})$.

$\sigma_{1}=$ Tensão principal maior na ruptura estimada para rocha intacta $(\mathrm{Pa})$.

$\boldsymbol{\rho}_{\mathbf{R}}=$ Densidade da rocha intacta $\left(\mathrm{g} / \mathrm{cm}^{3}\right)$.

Durante a detonação de uma carga de explosivos, um transdutor de vibração, posicionado em um ponto de observação, no campo muito próximo, não recebe contribuições de energia iguais de todas as porções da carga. Na prática, em situações na região do campo próximo, porções da carga distantes do sensor geram menos impacto que porções próximas.

Ryan e Harris (2000) propuseram a seguinte equação de atenuação das vibrações para o campo próximo (equação 3), cujo arranjo espacial das variáveis é aquele mostrado na Figura 1.

$\mathrm{PVS}=\mathrm{K}\left[\left(\frac{\ell}{\mathrm{R}_{0}}\right)\left(\phi-\arctan \left\{\frac{\mathrm{R}_{0} \tan \phi-\mathrm{H}}{\mathrm{R}_{0}}\right\}\right)\right]^{\alpha}$

onde:

PVS = Módulo do Vetor Velocidade de Vibração de Partícula Resultante (mm/s).

$\ell=$ Razão linear de carregamento $(\mathrm{kg} / \mathrm{m})$.

$\mathrm{H}=$ Comprimento da carga (m).

$\mathrm{R}_{0}=$ Distância horizontal do centro da carga ao transdutor (m).

$\phi=$ ângulo em radianos.

$\mathrm{K}$ e $\alpha=$ constantes do local.

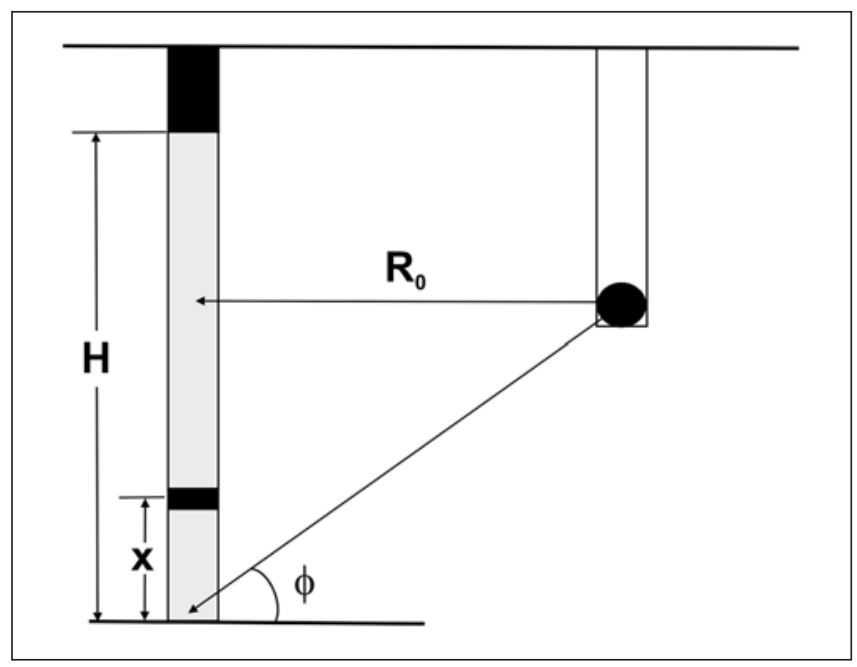

Figura 1 - Arranjo proposto para as variáveis da equação 3 (Ryan e Harris, 2000). 


\subsection{Metodologia utilizada para a obtenção da velocidade de propagação da onda primária $\left(\mathrm{V}_{\mathrm{p}}\right)$}

A velocidade de propagação da onda primária $\left(\mathrm{V}_{\mathrm{p}}\right)$ foi determinada através de sismografia, utilizando o arranjo mostrado na Figura 2.

Após a detonação de uma pequena carga, em um furo de $30 \mathrm{~cm}$, as 2 extremidades de dois fios AWG22, inseridas na carga, são rompidas. Com as 2 outras extremidades conectadas a um dispositivo “Auxiliar Trigger” de um sismógrafo de engenharia, inicia-se a contagem do tempo (t) necessário para a onda primária percorrer a distância (d) do furo até o sensor do sismógrafo.

Esse evento é registrado em um sismograma, que, através de análise no programa BlastWare III, permite a visualização do tempo, em relação ao zero da contagem, no qual a onda de vibração chega ao sensor (geofone) do sismógrafo. Assim, o cálculo da $V_{p}$ se faz através da seguinte expressão: $\mathbf{V}_{\mathbf{p}}=\mathbf{d} / \mathbf{t}$.

\subsection{Metodologia utilizada para avaliação da sobrequebra no contorno das galerias}

Para se averiguar a validade da utilização dos critérios de dano propostos, foram acompanhadas e medidas 4 frentes consecutivas, detonadas em uma galeria do nível 771. Após o cálculo dos raios das zonas de dano teóricas, estes foram comparados com o raio da zona de dano crítico levantada em torno das galerias, sendo esta aquela região situada entre a posição original dos furos de contorno (R1 na Figura 3) e a superfície final escavada (R2).

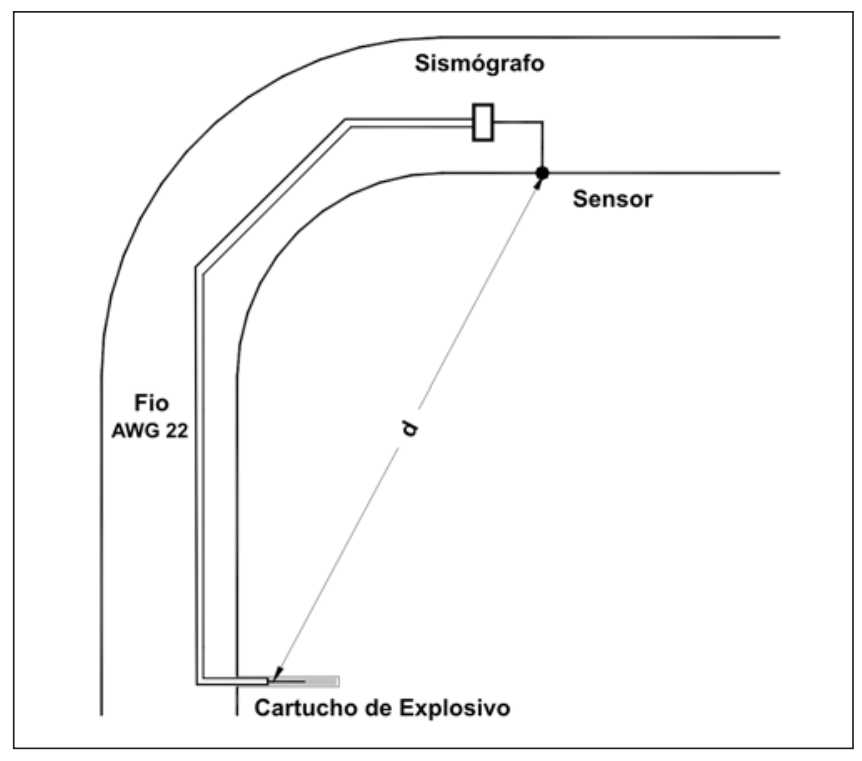

Figura 2 - Esquema para determinação da $V_{p}$
Para isto, foi desenvolvido um dispositivo simples, ao qual chamarei de MSL (Medidor de Sobrequebra Laser), que consiste de um distanciômetro Laser montado sobre um tripé fotográfico comum (Figura 4) e que, através de uma adaptação, permite que se leiam distâncias a partir do seu centro e a diferentes posições angulares, segundo qualquer plano do espaço (Figura 3).

\section{Resultados obtidos}

\subsection{Critérios de velocidade de partícula}

Os monitoramentos envolveram 2 classes de maciços rochosos: a encaixante Metariolito (Classe 2) e o minério Agalmatolito (Classe 2B).

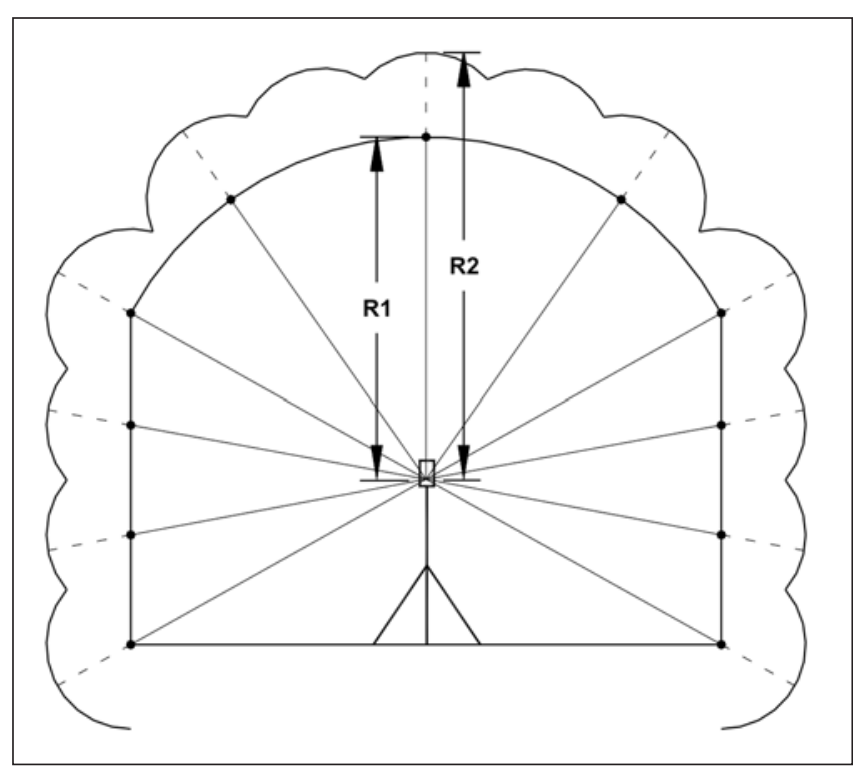

Figura 3 - Posição das visadas dos furos de contorno e da superfície final escavada, tomadas em um plano vertical perpendicular ao eixo da galeria.

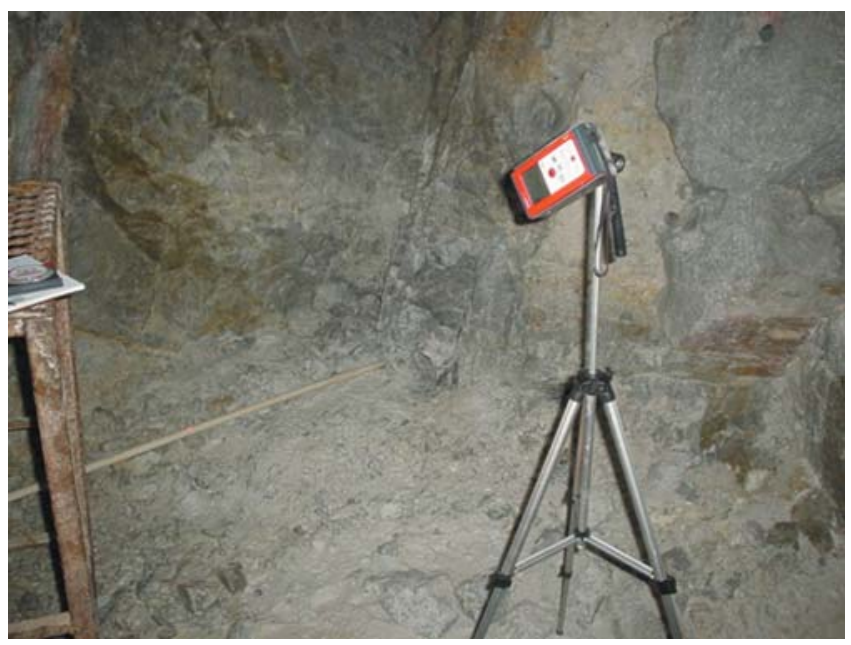

Figura 4 - MSL em posição para medida de furo de contorno. 
Avaliação dos danos causados pelo desmonte com explosivos no contorno de galerias subterrâneas...

Para o cálculo da PPV ${ }_{\text {crítica }}$ e da PPV mínima , substituíram-se os valores das propriedades geomecânicas dessas rochas, obtidas de ensaios laboratoriais (Fabiani et al., 2002), além dos valores médios das $\mathrm{V}_{\mathrm{p}}$ medidas. Os resultados constam da Tabela 1.

\subsection{Equação de atenuação da velocidade de partícula resultante de pico (PVS)}

A partir dos monitoramentos com sismógrafos de engenharia, foi elaborado o gráfico da Figura 5.

Assim, a equação de atenuação das vibrações, no campo próximo, adotada para a mina subterrânea da Lamil passa a ser:

PVS $=611,2\left[\left(\frac{\ell}{\mathrm{R}_{0}}\right)\left(\phi-\arctan \left\{\frac{\mathrm{R}_{0} \tan \phi-\mathrm{H}}{\mathrm{R}_{0}}\right\}\right)\right]^{0,5539}$

sendo os coeficientes locais: $\mathbf{K}=\mathbf{6 1 1 , 2}$ e $\boldsymbol{\alpha}=\mathbf{0 , 5 5 3 9}$.

Tabela 1 - Critérios de dano para cada maciço rochoso estudado.

\begin{tabular}{c|c|c}
\hline & \multicolumn{2}{|c}{ Tipo de maciço } \\
\hline Critério de Dano & Metariolito (Classe 2) & Agalmatolito (Classe 2B) \\
\hline $\operatorname{PPV}_{\text {crítica }}(\mathrm{mm} / \mathrm{s})$ & 2.098 & 718 \\
\hline $\operatorname{PPV}_{\text {mínima }}(\mathrm{mm} / \mathrm{s})$ & 552 & 567 \\
\hline
\end{tabular}

3.3 Determinação do raio de extensão das zonas de dano

Substituindo, na equação 4, os valores da PPV crítica $_{\text {e da }} P_{\text {mínima }}$, para cada uma das classes de maciço, e resolvendo-se a equação para o valor de $\mathrm{R}_{0}$ em um plano perpendicular ao eixo da carga, obtêm-se os raios das Zonas de Dano Crítico e Mínimo em torno dos furos de contorno. A Tabela 2 apresenta os valores assim calculados.

As Figuras 6 e 7 apresentam a representação espacial das zonas de dano em torno dos maciços de metariolito e agalmatolito.

\subsection{Avaliação da sobrequebra no contorno das galerias}

A Figura 8 mostra um exemplo dos levantamentos efetuados para comparação entre o raio de dano crítico teórico, calculado para o Metariolito, e os raios da zona danificada, em torno de frentes detonadas sucessivamente. As diferen-

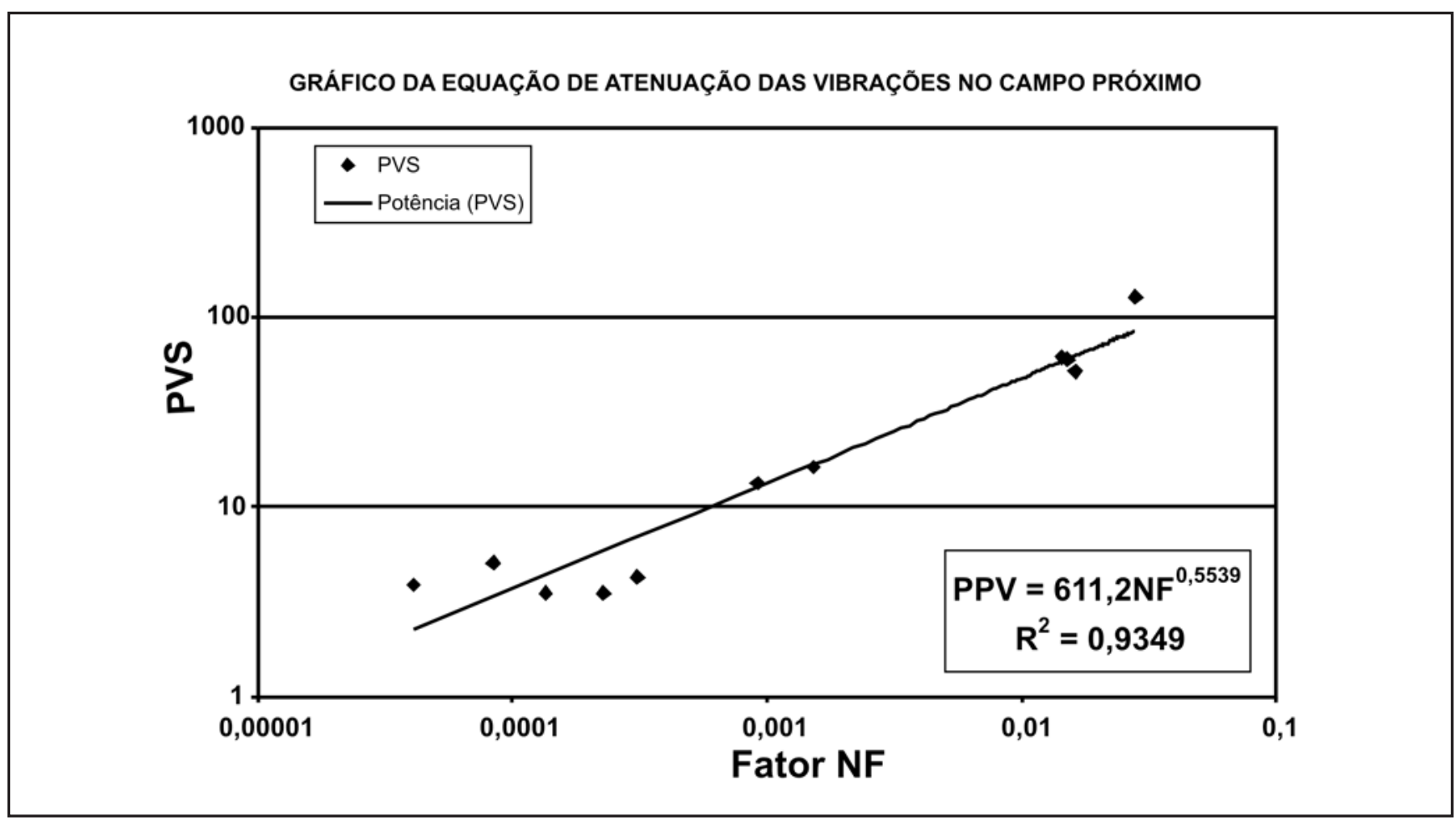

Figura 5 - Gráfico da regressão linear utilizada para a obtenção da equação de atenuação das vibrações no campo próximo. 
João Carlos Ribeiro Monteiro da Silva et al.

ças entre a posição original dos furos de contorno e a posição da superfície escavada foram tabeladas e os seus valores médios calculados para cada frente. Em média, os raios de dano efetivos foram 24\% menores que o raio de dano teórico, correspondendo a um erro de estimativa de $0,06 \mathrm{~m}$.

\section{Conclusão}

A comparação entre os valores dos critérios baseados na velocidade de partícula calculados e dos raios das zonas de dano teóricas, com aqueles constantes na literatura existente, demonstrou coerência com os resultados adotados por esses outros estudos.

Além disso, foi adotada metodologia, inédita no Brasil, para determinação da velocidade de propagação da onda primária $\left(\mathrm{V}_{\mathrm{p}}\right)$.
A comparação dos resultados dos levantamentos das superfícies escavadas com os valores estimados pelas equações teóricas desenvolvidas, no estudo, mostrou uma diferença que, considerando-se as muitas variáveis envolvidas no processo de cálculo dos raios de dano, pode ser considerada bastante aceitável. Isto reforça ainda mais a validade e aplicabilidade da metodologia adotada.

Tabela 2 - Raios das zonas de dano.

\begin{tabular}{c|c|c}
\hline & \multicolumn{2}{|c}{ Tipo de maciço } \\
\hline & $\begin{array}{c}\text { Metariolito } \\
\text { (Classe 2) }\end{array}$ & $\begin{array}{c}\text { Agalmatolito } \\
\text { (Classe 2B) }\end{array}$ \\
\hline $\begin{array}{c}\text { Raio da Zona de Dano Crítico } \\
(\mathrm{m})\end{array}$ & 0,24 & 1,29 \\
\hline $\begin{array}{c}\text { Raio da Zona de Dano } \\
\text { Mínimo (m) }\end{array}$ & 1,64 & 1,59 \\
\hline
\end{tabular}

\section{Referências bibliográficas}

1. CALDER, P. Perimeter blasting.In:Pit Slope Manual. Ottawa: CANMET (Canadá Centre for Mineral and Energy Technology), 1977. Cap. 7, p. 37.

2. FABIANI, P. A. A., QUADROS, E. F., BITAR, O. Y. Ensaios de compressão uniaxial com determinação do módulo de deformabilidade e coeficiente de

\section{Riolito Visualização das Zonas de Dano Teóricas}

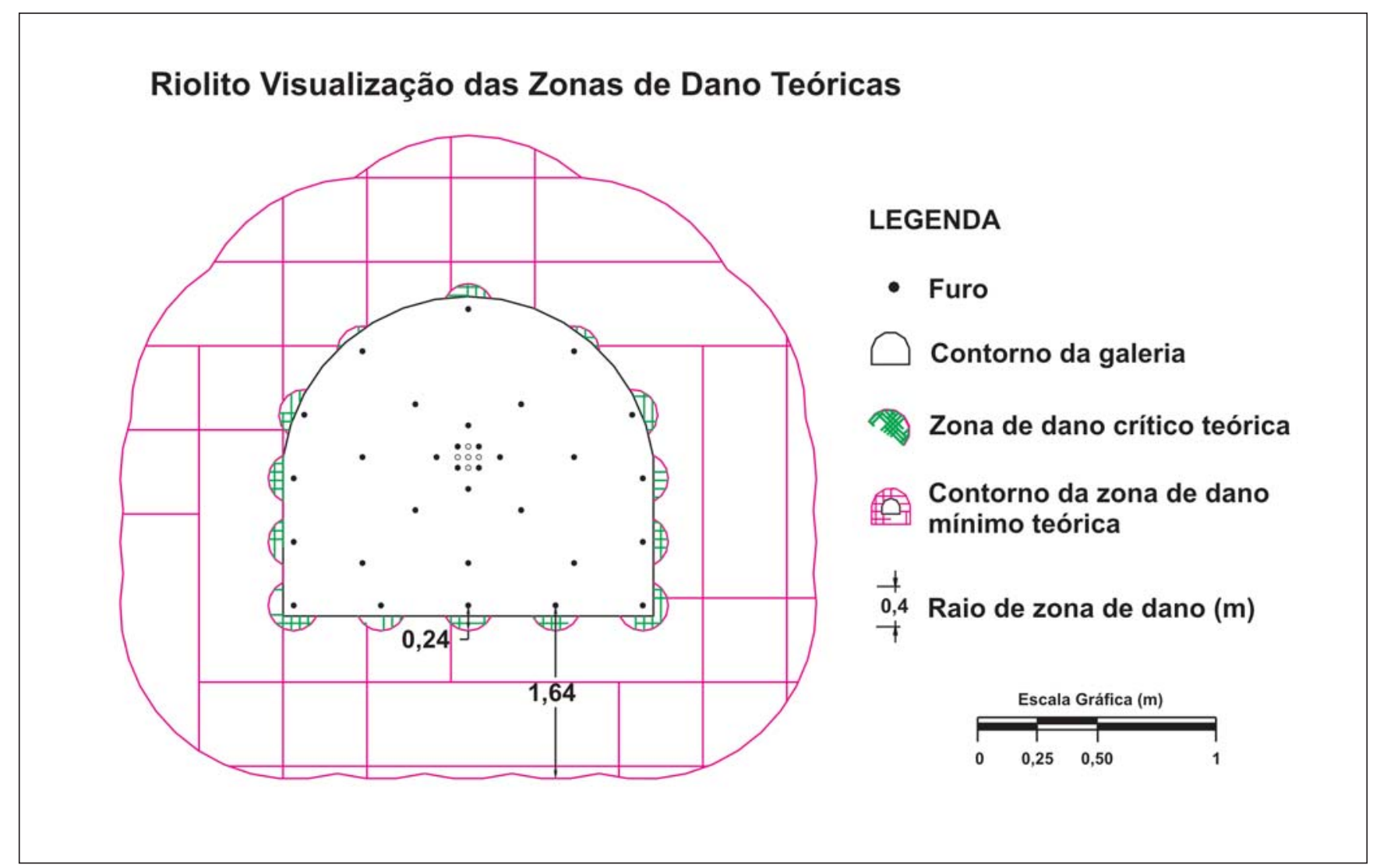

Figura 6 - Representação espacial das Zonas de Dano Crítico e Mínimo para galerias abertas em maciços de metariolito. 
Poisson, e ensaios para determinação indireta da resistência à tração em amostra de agalmatolito. São Paulo: IPT, 2002. Relatório técnico n. 61.408.

3. FABIANI, P. A. A., QUADROS, E. F., BITAR, O. Y. Ensaios de compressão uniaxial com determinação do módulo de deformabilidade e coeficiente de Poisson, e ensaios para determinação indireta da resistência à tração em amostra de metariolito. São Paulo: IPT, 2002. Relatório técnico n. 61.433.

4. PERSSON, P. A., HOLMBERG, R., LEE, J. Rock blasting and explosives engineering. Boca Raton: CRC Press, 1994. 540p.

5. RYAN, J. M., HARRIS, S. P. Using state of the art blast modeling software to assist the excavation of the Yucca Mountain nuclear waste repository. In: High-Tech Seminar. State-of-the-Art Blasting Technology Instrumentation and Explosives Applications, 2000. Proceedings. Orlando: Blasting Analysis International Inc., 2000. p. 407-423.

6. SILVA, J. C. R. M. As implicações do desmonte com explosivos na estabilidade da rocha remanescente em mina subterrânea. Ouro Preto: Escola de Minas, UFOP. 2004. p. 52-54. (Dissertação de Mestrado em Engenharia Mineral).

Artigo recebido em 13/06/2005 e aprovado em 11/01/2006.

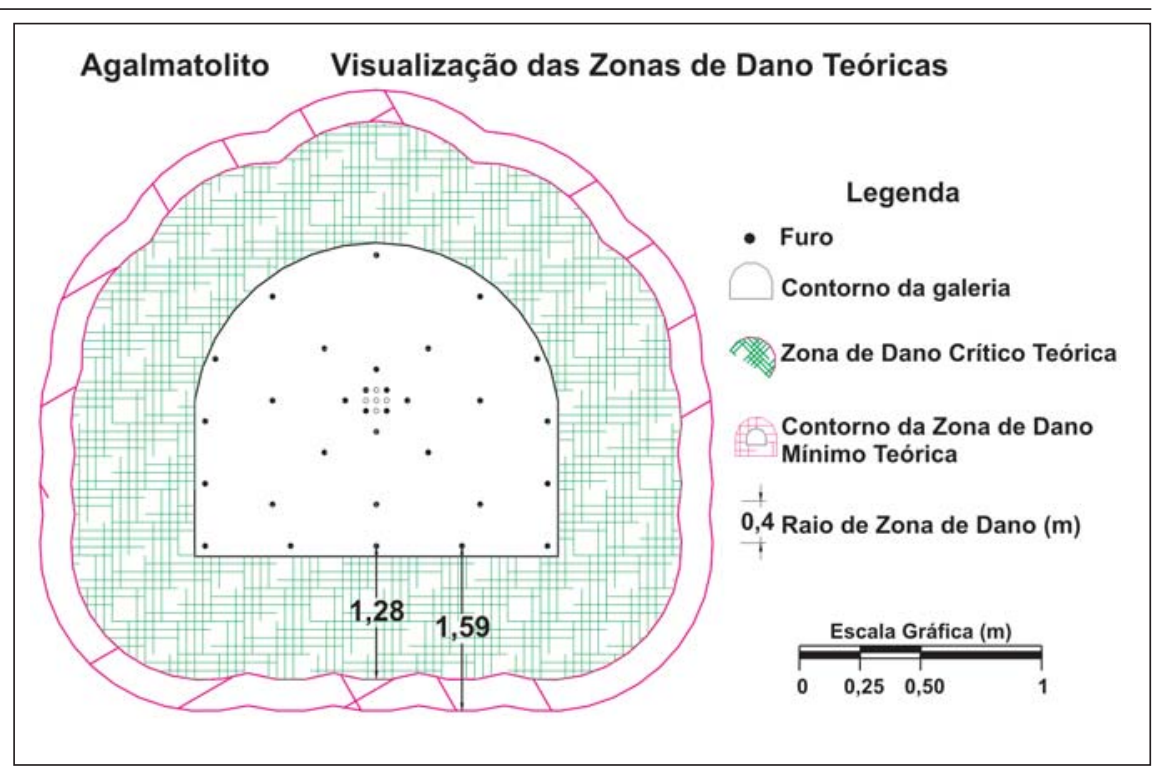

Figura 7 - Representação espacial das Zonas de Dano Crítico e Mínimo para galerias abertas em maciços de agalmatolito.

FRENTE 4 Visualização das Zonas de Dano Crítico

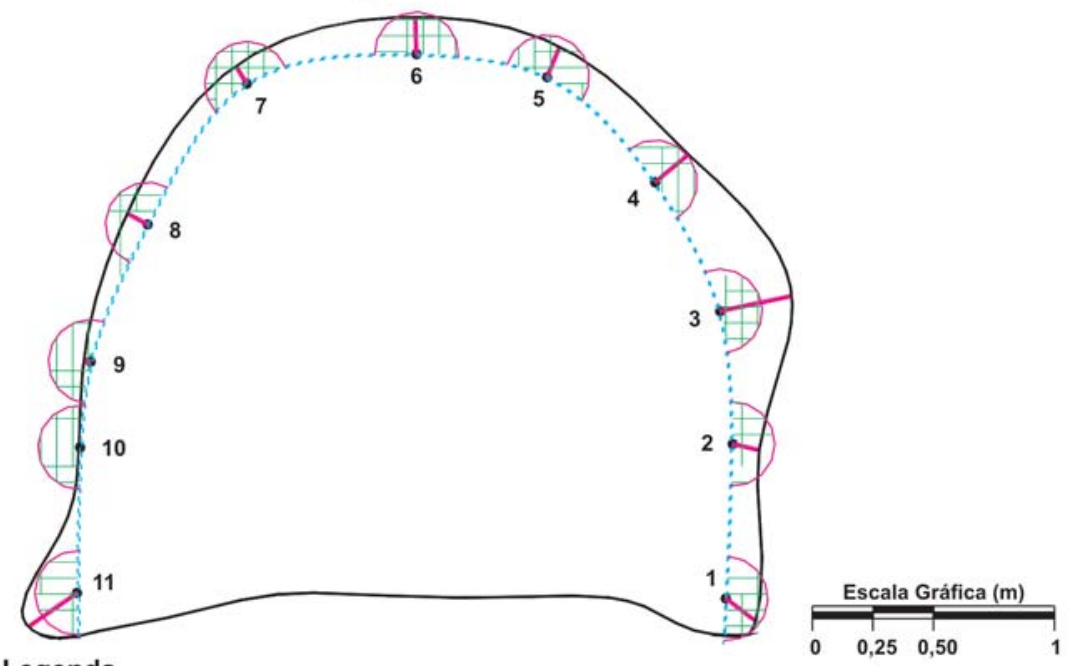

Legenda

Contorno da galeria projetado pelos furos

- Furo de contorno Número do furo

— Variação da posição do contorno após detonação

Zona de Dano Crítico Teórica

Contorno da Zona de Dano Crítico Medida

Figura 8 - Comparação visual entre os raios de dano teóricos para os furos de contorno da Frente 4 e os raios da zona danificada.

\section{www.rem.com.br 70 anos}

